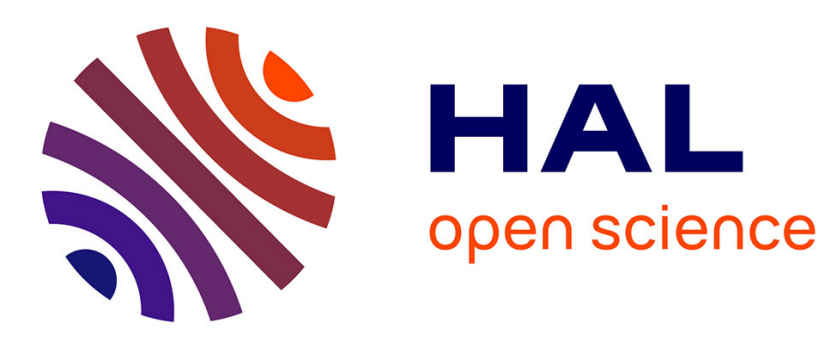

\title{
Les gilets jaunes et le triptyque " islam, banlieues, immigration": une machine à produire des fantasmes identitaires
}

Vincent Geisser

\section{- To cite this version:}

Vincent Geisser. Les gilets jaunes et le triptyque " islam, banlieues, immigration ": une machine à produire des fantasmes identitaires. Migrations Société, 2019. halshs-02317477

\section{HAL Id: halshs-02317477 \\ https://shs.hal.science/halshs-02317477}

Submitted on 17 Oct 2019

HAL is a multi-disciplinary open access archive for the deposit and dissemination of scientific research documents, whether they are published or not. The documents may come from teaching and research institutions in France or abroad, or from public or private research centers.
L'archive ouverte pluridisciplinaire HAL, est destinée au dépôt et à la diffusion de documents scientifiques de niveau recherche, publiés ou non, émanant des établissements d'enseignement et de recherche français ou étrangers, des laboratoires publics ou privés. 


\section{Éditorial}

\section{LES GILETS JAUNES ET LE TRIPTYQUE « ISLAM, BANLIEUES, IMMIGRATION » : UNE MACHINE À PRODUIRE DES FANTASMES IDENTITAIRES}

\section{Vincent Geisser}

Centre d'information et d'études sur les migrations internationales | "Migrations Société »

2019/1 N 175 | pages 5 à 16

ISSN 0995-7367

Article disponible en ligne à l'adresse :

https://www.cairn.inforevue-migrations-societe-2019-1-page-5.htm

Distribution électronique Cairn.info pour Centre d'information et d'études sur les migrations internationales.

(C) Centre d'information et d'études sur les migrations internationales. Tous droits réservés pour tous pays.

La reproduction ou représentation de cet article, notamment par photocopie, n'est autorisée que dans les limites des conditions générales d'utilisation du site ou, le cas échéant, des conditions générales de la licence souscrite par votre établissement. Toute autre reproduction ou représentation, en tout ou partie, sous quelque forme et de quelque manière que ce soit, est interdite sauf accord préalable et écrit de l'éditeur, en dehors des cas prévus par la législation en vigueur en France. Il est précisé que son stockage dans une base de données est également interdit. 


\section{Les gilets jaunes et le triptyque « islam, banlieues, immigration » : une machine à produire des fantasmes identitaires}

Vincent GEISSER

La grande majorité des sociologues, socio-historiens, anthropologues et politistes, malgré leurs divergences quant aux manières d'observer, aux méthodes d'analyse et aux cadrages théoriques des phénomènes sociaux, dues notamment à la différence de socialisation disciplinaire et de positionnement éthique dans le champ des sciences sociales, s'accordent pourtant à reconnaître que les gilets jaunes constituent un mouvement social protéiforme ${ }^{I}$. En somme, bien qu'il s'agisse d'une mobilisation protestataire relativement inédite dans l'histoire de la France contemporaine, les universitaires et les chercheurs en sciences sociales tendent à recourir à des variables sociologiques classiques, renvoyant notamment à l'inégalité de distribution des richesses, au sentiment d'injustice sociale et fiscale, aux vécus et aux ressentis des situations de domination dans les milieux populaires et au processus de désaffiliation partisane et syndicale observé depuis plusieurs années. Cette volonté d'historiciser - réintroduire le temps long — et de sociologiser privilégier les explications sociales - contraste avec les modes de décryptage identitaires qui se sont déployés dans les champs politique et médiatique, véhiculés par des acteurs partisans, des éditorialistes, des intellectuels et, parfois même, par des sympathisants et des compagnons de route $\mathrm{du}$ mouvement des gilets jaunes. Ces entreprises d'identitarisation du

1. HAYAT, Samuel, "Les gilets jaunes, l'économie morale et le pouvoir" [En ligne], 5 décembre 2018, https://samuelhayat.wordpress.com/2018/12/05/les-gilets-jaunes-leconomie-mora le-et-le-pouvoir/; MARLIÈRE, Éric, "Les 'gilets jaunes' vus par les quartiers populaires" [En ligne], Slate.fr, 9 janvier 2019, https://www.slate.fr/story/172035/gilets-jaunes-soutienquartiers-populaires; NOIRIEL, Gérard, "Le populaire dans tous ses états. Les gilets jaunes, l'antisémitisme et "la démocratie du public'" [En ligne], 20 février 2019 https://noiriel.word press.com/2019/02/20/les-gilets-jaunes-lantisemitisme-et-la-democratie-du-public/ ; PALHETA, Ugo, "Qui sont et que veulent les 'gilets jaunes' ? Entretien avec Benoît Coquard" [En ligne], Contretemps, 23 novembre 2018, https://www.contretemps.eu/sociologiegilets-jaunes/; GELDHOF, Frédéric, "De quoi le mouvement des gilets jaunes est-il le nom? Entretien avec Ahmed Boubeker" [En ligne], LeMuslimPost, 27 novembre 2018, https://lemuslimpost.com/gilets-jaunes-mouvement-rassemble-fragilites-sociales.html ; BERTHO, Alain, "II ne s'agit pas d'un simple mouvement social", Mediapart.fr, 8 décembre 2018 : https://www.mediapart.fr/journal/culture-idees/081218/alain-bertho-il-ne-s-agit-pas-d-unsimple-mouvement-social ; COLLECTIF, "'Gilets jaunes' : une enquête pionnière sur la 'révolte des revenus modestes'", Le Monde, 11 décembre 2018 ; TALPIN, Julien, "Les quartiers, les 'gilets jaunes' et la gauche", AOC, 15 février 2019, https://aoc.media/analyse/2019/ 02/15/quartiers-gilets-jaunes-gauche/. 
mouvement social se sont imposées dans l'espace public, autant pour le valoriser, cultivant un «Nous» imaginaire (la francité populaire face au mondialisme des élites), que pour le discréditer, dénonçant le retour en force au sein de la société française des identités meurtrières (antisémitisme, racisme, xénophobie, etc.). Sur ce point, nous partageons l'analyse de l'historien Gérard Noiriel sur les motivations latentes de certains acteurs dominants qui ont intérêt à légitimer des décryptages identitaires pour disqualifier les mobiles de la protestation : " Ce qu’ils [les gilets jaunes] déplorent le plus, ce sont les tentatives incessantes pour déplacer les problèmes de la sphère économique et sociale vers la sphère identitaire. C'est là que se situe en effet l'enjeu majeur de cette lutte sociale. Ceux qui ont hâte qu'on cesse de mettre en cause leurs privilèges cherchent à discréditer le mouvement en occupant le terrain identitaire où ils peuvent distribuer des leçons de morale à peu de frais. C'est cette stratégie qu'ont délibérément choisie Emmanuel Macron et son gouvernement $»^{2}$.

Toutefois, cette banalisation des lectures identitaires sur les origines et les motivations du mouvement des gilets jaunes ne relève pas simplement d'une entreprise de domination et/ou de manipulation des élites gouvernantes à l'égard de la colère des classes populaires. Nous apporterons ainsi quelques nuances par rapport à la thèse défendue par Gérard Noiriel. En effet, ces lectures identitaires nous paraissent à la fois plus profondes et plus complexes, répondant à des visées sociales contradictoires et parfois antagonistes.

Elles expriment d'abord la volonté de recréer une cohésion communautaire réelle ou imaginaire autour de valeurs communes (registre universaliste : égalité, fraternité, esprit de la Révolution de 1789 ou de la Commune de Paris, etc.) et aussi d'un noyau ethno-social fantasmé (registre particulariste : le pays réel, la France d'en bas) : les « vrais Gaulois populaires » opposés aux «élites européanisées et mondialisées ». Ce premier type de lecture identitaire est parfois présent chez certains acteurs du mouvement social mais ne fait pas forcément l'unanimité chez les gilets jaunes. Il est, en revanche, récurrent chez la majorité des éditorialistes, intellectuels et des leaders politiques nationalistes qui voient dans le mouvement des gilets jaunes la revanche sociale de la périphérie méprisée par les élites gouvernantes (la ruralité ruinée et la France des pavillons achetés à crédit) au profit de la périphérie assistée (les banlieues urbaines et immigrées).

À l'opposé de ce premier registre, les lectures identitaires participent aussi à une entreprise de délégitimation et de décrédibilisation des revendications sociales des gilets jaunes - nous partageons ici l'hypothèse de Gérard Noiriel - en les réduisant à leur dimension réactionnaire et

2. NOIRIEL, Gérard, "Le populaire dans tous ses états. Les gilets jaunes, I'antisémitisme et 'la démocratie du public'", art. cité. 
primaire : selon cette vision dépréciative, le mouvement des gilets jaunes ne constituerait que l'expression triviale et radicale des pulsions antisémites, islamophobes, racistes et xénophobes d'une "vieille France » qui est en train de mourir, le chant du cygne des franges populaires les plus réactionnaires de la société française. Ce type de décryptage a été très présent chez de nombreuses élites gouvernementales et de la majorité présidentielle (La République en marche), ainsi que chez des intellectuels et des éditorialistes se revendiquant des valeurs universalistes (la France républicaine et des Lumières), stigmatisant les gilets jaunes en les réduisant à leur identité réactionnelle et réactionnaire.

Mais plus fondamentalement, ces lectures identitaires du mouvement social des gilets jaunes se sont aussi immiscées, dans des modes de décryptage moins polémiques, chez les experts, universitaires et citoyens ordinaires qui ont cherché à comprendre "ce qui fait groupe », " ce qui fait mouvement », «ce qui fait rond-point» au-delà de la diversité des origines sociales, des particularismes locaux, des trajectoires socioprofessionnelles et des orientations politiques, religieuses et philosophiques des acteurs mobilisés. À l'instar de Monsieur Jourdain, nous avons tous contribué, à des degrés divers, à légitimer des décryptages identitaires du mouvement des gilets jaunes, parce que tout simplement nous cherchions à saisir son unité malgré l'hétérogénéité des acteurs protestataires, et ce d'autant plus que la sociologie des acteurs en lutte a largement évolué au fil des semaines. En ce sens, nous développerons l'hypothèse que la « charge identitaire », réelle ou fantasmée, du mouvement des gilets jaunes constitue moins une entreprise de manipulation qu'une coproduction - un phénomène partagé - des commentateurs, des observateurs, des chercheurs, des politiques (favorables ou hostiles) et des acteurs protestataires eux-mêmes, parce que le contexte social de la France des années 2000 conduit à «identariser» les phénomènes sociaux, même lorsqu'on veut y échapper. Le simple fait de répondre aux responsables politiques et aux médias que le mouvement des gilets jaunes ne saurait se réduire à la représentation caricaturale de la « revanche sociale » des classes populaires réactionnaires (blanches, rurales, sensibles aux sirènes de l'extrême droite), c'est déjà entrer dans un débat identitaire que l'on refuse par ailleurs.

Pour illustrer le caractère ambivalent et polysémique des décryptages identitaires du mouvement des gilets jaunes, nous porterons notre attention sur trois thématiques qui ont été à la fois récurrentes et saillantes dans le débat public : I) la polémique sur la visibilité/invisibilité des jeunes issus des banlieues urbaines sur les ronds-points et dans les manifestations de rue ; 2) la controverse sur la place de la religion en général, et de l'islam en particulier, dans les mobilisations des gilets jaunes ; 3) le positionnement, réel ou supposé, des acteurs protestataires sur la question de l'immigration, tantôt décrié comme un mouvement populiste et xénophobe, tantôt comme un mouvement laxiste et tiède sur la politique 
migratoire. Ces trois illustrations tendent à montrer que les lectures identitaires du mouvement des gilets jaunes ne sont le monopole ni de l'extrême droite, qui cherche à récupérer la protestation, ni des élites libérales qui lui sont majoritairement hostiles, mais qu'elles constituent un mode de décryptage récurrent chez les acteurs sociaux quelle que soit leur position par rapport aux mobilisations.

Comme le souligne avec ironie Ramsès Kéfi, journaliste à Libération, les regards sociaux sur les gilets jaunes révèlent une certaine obsession française à compter et à traquer les «banlieusards » au sein des rassemblements et des manifestations ${ }^{3}$. Nous pouvons ainsi relever dans la presse quotidienne et les chroniques des éditorialistes de nombreux commentaires consacrés à la présence/absence des jeunes de banlieues au sein des gilets jaunes: "Elles sont les grandes absentes du large mouvement de contestation qui soulève les quatre coins de la France depuis quinze jours. Pourtant concernées par les inégalités, le chômage et la baisse du pouvoir d'achat, les banlieues ne se sont jusqu'ici que peu associées aux revendications des gilets jaunes. Mais, à l'aube d'une troisième journée de mobilisation ce samedi, la France des "quartiers", comme on les appelle, pourrait, finalement, prendre le train de la révolte en marche »4. Ou encore: «Bien qu'a priori concernés au premier chef par les revendications des gilets jaunes, les habitants des quartiers populaires restent absents $d u$ mouvement $»^{5}$. Cette démarche de comptage ethnique n'est pas neutre sur le plan sociologi$q e^{6}$ : elle laisse transparaître des angoisses multiples et contradictoires sur la présence/absence des jeunes de banlieues ou, pour le dire plus crûment, sur la participation des Arabes, des Noirs et des musulmans à la protestation sociale, qui constituerait un «bon indicateur » de l'ouverture ou de la fermeture du mouvement et de ses "chances » de réussite ou d'échec: d'aucuns déplorent la faible visibilité des banlieusards ethnicisés au sein des gilets jaunes, parce qu'ils ne se sentiraient pas des « Français

3. KÉFI, Ramsès, "Gilets jaunes : 'Existe-t-il sur terre un compteur de banlieusards ?'" [En ligne], Libération.fr, 17 décembre 2018, https://www.liberation.fr/chroniques/2018/ 12/17/gilets-jaunes-existe-t-il-sur-terre-un-compteur-de-banlieusards_1698196.

4. BFM TV, "Gilets jaunes: les banlieues, grandes absentes du mouvement ?" [En ligne], 30 novembre 2018, https://www.bfmtv.com/societe/gilets-jaunes-les-banlieues-grandesabsentes-du-mouvement-1578008.html.

5. MORELLI, Sébastien; DEGRADI, Laurent; GARCIA, Florian, "Gilets jaunes : pourquoi le mouvement ne prend pas dans les cités" [En ligne], Le Parisien.fr, 24 janvier 2019, http://www.leparisien.fr/info-paris-ile-de-france-oise/gilets-jaunes-pourquoi-lemouvement-ne-prend-pas-dans-les-cites-24-01-2019-7996065.php.

6. À propos de cette injonction à la participation des banlieues, voir l'analyse pertinente du politiste Julien Talpin : « I/s ne sont pas là ! Depuis le début du mouvement, analystes, journalistes, sociologues, militants se demandent, comme une rengaine, où sont les quartiers populaires. Participent-ils au mouvement? Sont-ils solidaires? Comment répondre à ces questions sans énoncer une forme d'injonction à la participation qui ferait de nouveau passer les quartiers populaires pour des déserts politiques et leurs habitants pour des sauvageons ne pouvant qu'osciller entre business et religion, l'engagement collectif leur étant fondamentalement étranger? », in : TALPIN, Julien, "Les quartiers, les 'gilets jaunes' et la gauche", art. cité. 
comme les autres », d'autres, au contraire, craignent leurs tentatives d'infiltration dans la contestation sociale dans le but de la radicaliser, d'autres encore redoutent une possible jonction entre les périphéries rurales et les périphéries urbaines, une sorte de synergie diabolique des classes dangereuses qui mettrait à mal la République ${ }^{7}$.

Dans tous les cas, le thème de la visibilité/invisibilité des jeunes de banlieues au sein du mouvement des gilets jaunes obsède les commentateurs, les observateurs et les acteurs politiques français. Nous pouvons ici établir un parallèle avec l'obsession française à compter les musulmans dans les marches républicaines par solidarité avec les victimes du terrorisme $\left(l^{\prime} \ll\right.$ Après Charlie ») ${ }^{8}$, comme pour mieux souligner leur déficit de compassion patriotique et leur difficulté à s'identifier à la communauté nationale de deuil. Comme le note avec justesse le sociologue Hicham Benaissa ${ }^{9}$, ces populations sont souvent visibilisées dans leur invisibilité citoyenne, voire dans leur incivilité, et à l'inverse, invisibilisées dans leur visibilité civique, comme pour mieux mettre en évidence la distance sociale qui les sépare de la supposée majorité culturelle (les « vrais Français »).

Sans surprise, l'extrême droite et les penseurs nationalistes ont été les premiers à se saisir de cette thématique identitaire pour célébrer dans les gilets jaunes l'incarnation de la « France populaire », oubliée et méprisée par les politiques, en l'opposant systématiquement au mondialisme et au multiculturalisme des jeunes de banlieues (la culture rap, Snapchat et Mac Do) qui seraient l'objet de toutes les attentions de l'action publique. On peut lire ainsi sur le site d'extrême droite Riposte laïque un décryptage sociologique très orienté de la mobilisation des gilets jaunes, opérant une distinction entre les bons manifestants issus du «petit peuple de France » et les mauvais manifestants représentés par «les racailles» et les «casseurs » originaires des banlieues des grandes agglomérations : «En parcourant pendant des heures les différents terrains d'affrontement, à Paris, durant ces trois journées, voici ce que j'ai vu et entendu. J'ai vu monter dans les avenues de la capitale le peuple de France, le petit peuple, ceux qui font marcher la machine... Ils allaient d'un pas alerte, le plus souvent en groupe, parfois en troupe, même si l'on remarquait des isolés, en nombre. [...] Les racailles, pour leur part, venaient pousser à l'insurrection, au désordre, le mettant à

7. Sur le fantasme de la jonction entre banlieues rurales et banlieues urbaines, voir l'article critique du sociologue SIDI MOUSSA, Nedjib, "Gilets jaunes et banlieues françaises : une convergence impossible ?" [En ligne], Middleeasteye.net, 19 janvier 2019, https://www.middleeasteye.net/fr/opinion/gilets-jaunes-et-banlieues-francaises-uneconvergence-impossible.

8. GEISSER, Vincent; MARONGIU-PERRIA, Omero; SMAÏL, Kahina, Musulmans de France : la grande épreuve. Face au terrorisme, Ivry sur Seine : Les éditions de I'Atelier, 2017, 311 p.

9. BENAISSA, Hicham, "L'injonction paradoxale à l'islam de France" [En ligne], Libération.fr, 12 janvier 2015, https://www.liberation.fr/societe/2015/01/12/I-injonction-paradoxale-aI-islam-de-france_1179288. Sur le même thème, voir aussi I'article de ROY, Olivier, "La peur d'une communauté qui n'existe pas", Le Monde, 9 septembre 2015. 
profit pour casser et piller. Leur "uniforme" les identifie aussi : casquette ou bonnet, tour de cou ou passe-montagne, blouson et jean collant ou pantalon de survêt, le tout de couleur noire ou gris foncé, et des "baskets" aux pieds. Ils tentent bien sûr, le plus souvent, de dissimuler leur faciès... Ceux-ci, m'a-t-il semblé, vont rarement au contact des forces de l'ordre, ils préfèrent agir sur les points faibles du dispositif pour casser, brûler et piller. Ils semblent pour une bonne part, tout comme les black blocs d'ailleurs, plutôt allergiques au gilet jaune, mais si celui-ci leur permet de manouvrer plus sereinement, il sort sans doute du sac assez rapidement... Ils agissent aussi toujours en bandes de quatre à quinze individus ${ }^{\mathrm{IO}}{ }^{\mathrm{O}}$. Le journal de l'ultra-droite nationaliste Rivarol développe un décryptage identitaire assez similaire du mouvement des gilets jaunes, véhiculant une xénophobie et un antisémitisme à peine voilés : "Nous avons affaire, avec les gilets jaunes, à des Français de base : nul Malika, Akim, Shlomo, N'Golo ou Abdel dans leurs rangs, mais des quidams bien de chez nous, loin des hordes de racailles venus du 93 pour faire leurs courses de Noël à Paris, en fin de journée, les quatre premiers samedis de décembre ${ }^{\text {II }}$. Dans un registre plus nuancé, mais tout aussi caricatural sur le plan sociologique, Alain Finkielkraut oppose la France périphérique délaissée par les élites qui constituerait, selon lui, le cœur du mouvement des gilets jaunes, aux banlieues urbaines «sur-assistées » par les politiques publiques: "Les classes politiques et médiatiques n'en avaient que pour la banlieue alors que la France périphérique était de plus en plus précaire ${ }^{\mathrm{I2}}$. Pour le philosophe, le mouvement des gilets jaunes exprime la revanche sociale légitime des « petits blancs » méprisés par trente ans de politiques multiculturelles. Toutefois, après son agression par un gilet jaune lors de la mobilisation du I6 février 20I9, le philosophe changera radicalement sa position en considérant que le mouvement est devenu une $«$ secte $»^{13}$.

Ces catégories identitaires opposant sur un mode manichéen les couples périphéries rurales/banlieues urbaines, classes populaires françaises/classes populaires immigrées, Français de souche délaissés/banlieusards assistés ont été, en grande partie, déconstruites par les premières études sociologiques

10. DUBOIS, Jean, "Gilets jaunes, racailles et casseurs : ce que j'ai vu lors de trois manifs parisiennes..." [En ligne], Riposte laïque.com, 27 décembre 2018, https://riposte laique.com/gilets-jaunes-racailles-et-casseurs-ce-que-jai-vu-lors-de-trois-manifsparisiennes.html.

11. Extrait de Rivarol du 9 janvier 2019, cité par SIDI MOUSSA, Nedjib, "Gilets jaunes et banlieues françaises : une convergence impossible ?" [En ligne], Middleeasteye.net, 19 janvier 2019, https://www.middleeasteye.net/fr/opinion/gilets-jaunes-et-banlieues-fran caises-une-convergence-impossible.

12. Cité par DEVILLERS, Sonia, "Ces médias qui ne trouvent pas la banlieue parmi les gilets jaunes" [En ligne], France Inter, 3 décembre 2018, https://www.france inter.fr/emissions/I-edito-m/I-edito-m-03-decembre-2018.

13. Cité par BARRACO, Florent; DURGET, Emmanuel, "Alain Finkielkraut : 'les gilets jaunes sont devenus une secte'" [En ligne], Le Point.fr, 19 février 2019, https://www.le point.fr/debats/alain-finkielkraut-les-gilets-jaunes-sont-devenus-une-secte-19-02-20192294372_2.php. 
parues sur le mouvement des gilets jaunes ${ }^{14}$. Faute de recul suffisant, leurs auteurs restent généralement très prudents dans leurs conclusions. Toutefois, ils remettent en cause l'idée reçue selon laquelle les banlieusards « ethnicisés » auraient été absents, voire auraient carrément boycotté les mobilisations des gilets jaunes. Leurs études montrent, au contraire, que la participation est très variable selon les contextes locaux, les milieux sociaux (le monde des banlieues n'est pas homogène), les profils socioprofessionnels des individus, les sympathies politiques et aussi la temporalité du mouvement. Sans verser dans l'image idyllique d'un ralliement massif des jeunes des banlieues au mouvement des gilets jaunes, les enquêtes sociologiques permettent de déconstruire les lectures ethnicisantes de la protestation sociale et, au final, de remettre en cause les catégories essentialistes et réifiantes «jeunes de banlieues» ou «jeunes issus de l'immigration » qui relèvent davantage du discours politico-médiatique et du sens commun que de l'analyse sociologique. Le sociologue Éric Marlière note ainsi que "certains médias ont certes noté l'absence des gens ou des jeunes de banlieues dans les marches impulsées par les syndicats ou par les "gilets jaunes". Mais dans les cortèges figurent bien des personnes actives issues des quartiers, syndiquées, qui ne se mobilisent pas comme "issues de..." mais tout simplement en tant que travailleurs et travailleuses $»^{15}$.

Les banlieusards issus des cités urbaines ont donc rejoint le mouvement des gilets jaunes, en prenant part aux manifestations de rues ou à l'occupation des ronds-points, à partir de prédispositions personnelles à agir et à se mobiliser, mais rarement sur le mode de l'engagement collectiff $^{16}$, à l'exception du collectif Justice et vérité pour Adama Traoré, du Front uni des immigrations et des quartiers populaires (FUIQP), du Collectif banlieues respect ou encore de quelques groupes de militants locaux issus de quartiers populaires: "Tant qu'une partie de la population subira les violences institutionnelles, il ne sera pas possible d'en finir avec un système qui broie des vies. Nous sommes tous des gilets jaunes, les quartiers sont en première ligne de la précarité. Saisissons cette occasion historique et unissons nos forces! Nous sommes investis depuis plusieurs semaines au sein du mouvement des gilets jaunes. Poursuivons l'alliance, gilets jaunes, quartiers populaires en banlieue et zones rurales, travailleurs avec ou sans emploi, avec ou

14. COLLECTIF, '”Gilets jaunes' : une enquête pionnière sur la 'révolte des revenus modestes', art. cité ; SEBBAH, Brigitte ; SOUILLARD, Natacha ; THIONG-KAY, Laurent ; SMYRNAIOS, Nikos, Les gilets jaunes, des cadrages médiatiques aux paroles citoyennes [En ligne], Rapport de recherche préliminaire, Toulouse: LERASS, 26 novembre 2018, https://fr.scribd.com/docu ment/394250648/Rapport-Gilets-Jaunes.

15. MARLIÈRE, Éric, "Les 'gilets jaunes' vus par les quartiers populaires", art. cité.

16. PALHETA, Ugo, "Qui sont et que veulent les 'gilets jaunes'? Entretien avec Benoît Coquard", art. cité ; GELDHOF, Frédéric, "De quoi le mouvement des gilets jaunes est-il le nom? Entretien avec Ahmed Boubeker", art. cité. 
sans papiers, jeunesse précarisée, tous ensemble $! »^{17}$. Cependant, ce projet utopique d'un engagement collectif des banlieues avec ou aux côtés des gilets jaunes a fait l'objet de nombreux débats contradictoires parmi les militants issus des quartiers qui ont généralement développé des points de vue contrastés, voire opposés, sur le mouvement, à l'instar du Parti des indigènes de la République (formé principalement par des individus des classes moyennes ayant quitté la banlieue depuis de nombreuses années) qui entend appuyer les revendications des gilets jaunes, tout en considérant que c'est d'abord un mouvement de "prolétaires blancs » : "En effet, si les classes populaires blanches se révoltent depuis plus deux mois, c'est aussi parce qu'elles ont les moyens de le faire: non pas les moyens matériels, mais les moyens symboliques. Nous l'avons déjà dit, il est plus injuste et illégitime d'être un pauvre blanc qu'un pauvre non blanc. Étant chez lui, le blanc pauvre se verra attribuer une plus grande légitimité à contester son sort, et cela, y compris de manière violente. C'est ainsi qu'il faut comprendre les discours dans lesquels les gilets jaunes expliquent qu'ils sont "juste" des "Français normaux", "d'honnêtes travailleurs" ne parvenant plus à boucler leur fin de mois. La signification de ce type de propos est simple : ils méritent d'autant moins d'être pauvres qu'ils sont français et travailleurs. Leur appartenance à la Nation n'est jamais remise en cause, et c'est parce qu'ils se sentent pleinement français et sont considérés comme tels qu'ils pensent être légitimes à réclamer justice, à réclamer leur dû et à contester. Leur blanchité leur garantit également un large soutien dans la population, quand bien même ils ont recours à des actions violentes et qu'ils dégradent des lieux hautement symboliques comme l'Arc de Triomphe $»^{18}$.

Si la question de la présence/absence des jeunes de banlieues au sein du mouvement des gilets jaunes constitue une machine à produire des fantasmes identitaires tous azimuts, celle de la place de la religion dans la mobilisation contribue à les décupler: les décryptages identitaires du mouvement ont parfois convergé avec les représentations anxiogènes sur l'islamisation de la société française ${ }^{19}$ en général, et des milieux populaires en particulier. En effet, les porte-parole de l'extrême droite et de la droite identitaire ont contribué à répandre l'idée selon laquelle les musulmans avaient très largement boycotté le mouvement des gilets jaunes parce qu'ils ne se sentiraient pas français et qu'ils rejetteraient massivement les valeurs judéo-chrétiennes du «petit peuple». On peut lire ainsi sous la plume de Christine Tassin ${ }^{20}$, ancienne professeure de lettres et principale

17. COLLECTIF, "Stop aux violences policières : des quartiers populaires aux gilets jaunes !" [En ligne], 29 janvier 2019, https://paris-luttes.info/stop-aux-violences-policieres-des- 11580.

18. XELKA, Wissam, "'Quartiers populaires' et gilets jaunes : mêmes galères même combat ?" [En ligne], Parti des Indigènes de la République, 30 janvier 2019, http://indigenesrepublique.fr/quartiers-populaires-et-gilets-jaunes-memes-galeres-meme-combat/.

19. LIOGIER, Raphaël, Le mythe de l'islamisation : essai sur une obsession collective, Paris : Éd. du Seuil, 2012, 224 p.

20. COROLLER, Catherine, "'Bouffeuse d'islam', portrait de Christine Tassin", Libération.fr, 2 mars 2011, https://www.liberation.fr/societe/2011/03/02/bouffeuse-d-islam_718571. 
animatrice du site Riposte laïque : "Quelles que soient les raisons que vous avez cochées, il n'y a qu'une conclusion à tirer de tout cela : les musulmans qui se mettent à l'écart d'un mouvement de masse comme celui des gilets jaunes montrent qu'ils ne se sentent pas français, qu'ils ne sont pas français, ce qui démontre ô combien ce que nous affirmons depuis des lustres : l'islam est incompatible avec la France, avec la République, avec les Français $»^{2 \mathrm{I}}$. Le journal en ligne Boulevard Voltaire, proche de l'extrême droite et de la droite identitaire, va jusqu'à développer une théorie de la «tripartition du corps social » aux accents très maurassiens (la théorie des quatre États confédérés: protestants, juifs, francs-maçons et métèques), expliquant que le mouvement des gilets jaunes viendrait révéler une fracture profonde entre trois entités sociales, à savoir la classe dirigeante, les classes populaires et moyennes françaises de souche paupérisées et la classe immigrée musulmane : "On peut plus aisément y voir l'expression de la "tripartition" du corps social avec, d'un côté, la classe dirigeante et tous ceux qui vivent directement ou indirectement du système sans trop souffrir encore, d'un autre côté les laisséspour-compte de la France périphérique et des classes moyennes et populaires paupérisées et, enfin, la France des immigrés, cimentée idéologiquement par l'islam, ne se sentant pas concernée par les déboires des seconds et vivant largement sous perfusion des crédits publics octroyés par les premiers $»^{22}$.

Toutefois, le mythe de l'islamisation des classes populaires est aussi polymorphe et réversible. Plutôt que de déplorer l'absence (ou le boycott) des musulmans du mouvement des gilets jaunes, certains essayistes, polémistes et intellectuels se sont employés à dénoncer les risques d'infiltration islamique dans la protestation sociale. Selon eux, l'évolution du mouvement des gilets jaunes révélerait une entreprise de récupération de la part des islamo-gauchistes, des antisionistes, des antisémites et même des Frères musulmans qui saisiraient l'opportunité d'asseoir leur hégémonie idéologique sur les classes populaires en général, et sur les banlieues urbaines en particulier ${ }^{23}$. Cette thèse totalement fantasmatique a notamment été développée par le philosophe Michel Onfray, qui analyse le mouvement des gilets jaunes comme l'expression de la «coalition des haineux »: "Or, il existe deux récupérations des gilets jaunes dont on parle peu : la récupération par la gauche et la récupération par les islamistes. [...] Examinons la récupération par un certain islam. Le compagnonnage entre

21. TASSIN, Christine, "Gilets jaunes : mais où étaient donc les musulmans le 17 novembre ?" [En ligne], Riposte laïque.com, 19 novembre 2018, https://ripostelaique.com/gilets-jaunesmais-ou-etaient-donc-les-musulmans-le-17-novembre.html.

22. "Immigration, islam et gilets jaunes : un des non-dits du mouvement de contestation" [En ligne], Boulevard Voltaire.fr, 8 janvier 2019, http://www.bvoltaire.fr/immigration-islam-etgilets-jaunes-un-des-non-dits-du-mouvement-de-contestation/.

23. LOUIZI, Mohamed, "Gilets jaunes et Alexandre Benalla : I'ours islamiste est sorti de ses banlieues (les preuves)" [En ligne], 29 décembre 2018, http://mohamed louizi.eu/2018/12/29/gilets-jaunes-et-alexandre-benalla-lours-islamiste-est-sorti-deses-banlieues-les-preuves/. 
une gauche anticapitaliste, donc antisioniste, de fait antisémite, et un islam venu des banlieues, est avéré. Il signe la collusion entre l'anticapitalisme et l'islamisme. Ce mélange est explosif. Il est en passe d'emporter le mouvement des gilets jaunes. Ceux qui s'en réjouissent, Macron et les siens, ont bien tort. Car, dans ses suites, pareille convergence des luttes n'emporterait pas seulement les gilets jaunes, elle ouvrirait également la voie à une libération de la violence dont le pouvoir, déjà débordé par beaucoup moins, ne saurait que faire... Ce compagnonnage du jaune et du vert était déjà visible le jour des dégradations effectuées à l'Arc de Triomphe [...]. Dans la mouvance salafiste, du côté des Frères musulmans, dans les pays comme le Qatar, le mouvement des gilets jaunes est perçu comme une occasion de faire tomber l'Occident pensé comme judéo-chrétien, donc comme un adversaire rabique. Une fois tombé l'édifice, il ne resterait plus qu'à y installer une idéologie nouvelle ${ }^{24}$. Cette thèse de l'islamisation des gilets jaunes a d'ailleurs trouvé un début de légitimation scientifique dans les analyses du politiste Gilles Kepel, spécialiste reconnu des mouvements musulmans en France et dans le monde: "Les sites djihadistes se réjouissent, car ils perçoivent que la France, elle aussi, est entrée dans le chaos. Pour eux, ça montre que la démocratie est fichue. On voit un tract défiler sur les web-télés des djihadistes français en Syrie: ils ont repris quelque chose sur l'Arc de Triomphe qu'ils avaient déjà fait et ont ajouté quelques gilets jaunes dessus. Ils expliquent qu'il faut désormais faire des actions spectaculaires en France, parce que ça aura plus d'impact qu'ailleurs $»^{25}$. Dans le cadre de cet éditorial, il ne nous appartient pas de juger de la validité scientifique de ces thèses mettant en scène le boycott musulman ou l'infiltration islamique du mouvement des gilets jaunes, mais nous pouvons d'ores et déjà souligner la faiblesse des éléments empiriques sur lesquels elles s'appuient (absence d'enquête sociologique sérieuse) ainsi que leur tendance à essentialiser les attitudes et les comportements des jeunes issus des banlieues (catégorie déjà critiquable) en les enfermant systématiquement dans leur « identité islamique ».

Enfin, dernière thématique de cette machine à produire des fantasmes identitaires : les positions, réelles ou supposées, des gilets jaunes sur la question de l'immigration. Sur un sujet déjà fortement passionnel au sein de l'espace public, les décryptages identitaires de la protestation sociale se sont donnés libre cours. En premier lieu, la présidence de la République, les milieux gouvernementaux et certains journalistes et éditorialistes hostiles au mouvement social ont cherché à présenter les gilets jaunes comme une protestation réactionnaire et passéiste, mue par des valeurs xénophobes, anti-européennes et surtout anti-immigrés. Les polémiques

24. ONFRAY, Michel, "Le Jaune et le Vert" [En ligne], 2019: https://michelon fray.com/interventions-hebdomadaires/le-jaune-le-vert.

25. "Pour Gilles Kepel, 'les djihadistes se réjouissent' du mouvement des gilets jaunes" [En ligne], Le Point.fr, 15 décembre 2018, https://www.lepoint.fr/art-de-vivre/pour-gilleskepel-les-djihadistes-se-rejouissent-du-mouvement-des-gilets-jaunes-15-12-20182279536_4.php. 
autour du Pacte de Marrakech sur les migrations ${ }^{26}$, la dénonciation de sans-papiers par des gilets jaunes dans la Somme (affaire du barrage de Flixecourt $)^{27}$ ou la suspicion de la récupération du mouvement par le Rassemblement national de Marine Le Pen et les Identitaires ${ }^{28}$ ont été très largement véhiculées dans les champs politique et médiatique afin de discréditer la protestation sociale, alors que les enquêtes sociologiques montrent que les positions des gilets jaunes sur les questions migratoires sont à la fois plus nuancées ${ }^{29}$ et surtout plus diversifiées en fonction des classes d'âge, des niveaux d'études, des statuts socioprofessionnels, des lieux de résidence, etc. ${ }^{30}$. Il est vrai que les activistes du groupe Génération identitaire ${ }^{3 \mathrm{I}}$ et les polémistes d'extrême droite ont très largement contribué à renforcer cette image des gilets jaunes comme un mouvement fondamentalement hostile à l'immigration, parce que composé très majoritairement de « vrais Français » lâchés par des politiques gouvernementales réputées pro-immigration. Une fois encore, le thème de l'absence (ou le boycott) des enfants de l'immigration des cortèges des gilets jaunes a été pointé du doigt par les éditorialistes d'extrême droite et des milieux nationalistes, comme pour mieux mettre en exergue leur déficit d'intégration à la nation: "Quant aux Français issus de l'immigration, ils brillent par leur absence : ça en dit long sur leur implication dans la société et ses luttes sociales. Les fameuses minorités invisibles le sont vraiment ici : nos prétendues "chances pour la France" le sont sûrement pour le pouvoir, qu'elles n'ont pas l'air de contester beaucoup. On voit bien le danger des conséquences

26. "Immigration. Qu'est-ce que le 'Pacte de Marrakech', approuvé ce lundi matin ?" [En ligne], Le Dauphiné Libéré.fr, 6 décembre 2018, https://www.ledauphine.com/francemonde/2018/12/06/qu-est-ce-que-le-pacte-de-marrakech-que-doit-signer-macron-lundi.

27. TÉSORIÈRE, Ronan, "Quand des gilets jaunes dénoncent des migrants aux gendarmes" [En ligne], Le Parisien.fr, 21 novembre 2018, http://www.leparisien.fr/faits-divers/quand-desgilets-jaunes-remettent-des-migrants-aux-gendarmes-21-11-2018-7948308.php.

28. "Le RN et Marine Le Pen pas si représentatifs des gilets jaunes (sondage)" [En ligne], France Soir.fr, 12 mars 20919, http://www.francesoir.fr/politique-france/le-rn-et-marine-lepen-pas-si-representatifs-des-gilets-jaunes-sondage.

29. Dans la plate-forme revendicative contenant 42 propositions établies par certains militants du mouvement des gilets jaunes, on peut lire : Proposition $n^{\circ} 18$ : Que les causes des migrations forcées soient traitées; Proposition $n^{\circ} 19$ : Que les demandeurs d'asile soient bien traités. Nous leur devons le logement, la sécurité, l'alimentation ainsi que l'éducation pour les mineurs. Travaillez avec l'ONU pour que des camps d'accueil soient ouverts dans de nombreux pays du monde, dans l'attente du résultat de la demande d'asile; Proposition $n^{\circ} 20$ : Que les déboutés du droit d'asile soient reconduits dans leur pays d'origine ; Proposition $n^{\circ} 21$ : $Q u^{\prime} u n e$ réelle politique d'intégration soit mise en œuvre. Vivre en France implique de devenir français (cours de langue française, cours d'Histoire de la France et cours d'éducation civique avec une certification à la fin du parcours). Extraits tirés de "Liste des 42 revendications des gilets jaunes" [En ligne], 2 décembre 2018, https://blogs.media part.fr/jeremiechayet/blog/021218/liste-des-42-revendications-des-gilets-jaunes.

30. COLLECTIF, "'Gilets jaunes' : une enquête pionnière sur la "révolte des revenus modestes", art. cité ; SEBBAH, Brigitte ; SOUILLARD, Natacha ; THIONG-KAY, Laurent ; SMYRNAIOS, Nikos, Les Gilets Jaunes, des cadrages médiatiques aux paroles citoyennes, op. cit.

31. "Génération Identitaire enfile son gilet jaune !" [En ligne], 24 novembre 2018, https://generationidentitaire.org/2018/11/22/manifestations-du-24-novembre-gene ration-identitaire-enfile-son-gilet-jaune/. 
de l'immigration, qui produit l'isolement communautaire, la fracture du peuple français et l'affaiblissement relatif des luttes revendicatives. Au moment où l'on observe une alliance, dans la rue, du nouveau prolétariat et de la nouvelle petite bourgeoisie, les Français d'origine immigrée rompent l'unité populaire. La prochaine étape sera-t-elle l'instrumentalisation des absents pour des affrontements communautaires ? $\$^{32}$. Mais la "machine à fantasmes identitaires » a aussi fonctionné en sens inverse pour souligner la tiédeur, voire le laxisme, des gilets jaunes en matière d'immigration et de contrôle des frontières, suspectés de s'être laissés " gauchir » au fil des semaines de protestation ${ }^{33}$.

Depuis le début du mouvement social, en octobre 20I8, les observateurs, commentateurs et politiques ont beaucoup parlé de «crise des gilets jaunes », comme s'ils cherchaient à faire porter la responsabilité du désordre social et sécuritaire sur les acteurs de la protestation. Et précisément, l'un des procédés récurrents utilisés pour retourner les « causes » du malaise social a consisté à faire marcher la machine à fantasmes identitaires autour des thématiques anxiogènes désormais classiques islam, antisémitisme/antisionisme, immigration et banlieues -, contribuant à jeter un voile sur la question sociale. Mais contrairement à une idée reçue, cette machine à fantasmes n'est pas le propre des politiques, des élites gouvernantes et des faiseurs d'opinion : elle a fonctionné dans tous les milieux sociaux sans exception, y compris chez ceux qui prétendent réintroduire de la distance et de la rationalité dans le débat public, au point de rendre incompréhensibles des revendications sociales pourtant « simples»: dignité et humanité. C'est donc moins de «crise des gilets jaunes » qu'il conviendrait de parler que de « crise de lisibilité » du monde social, de plus en plus parasité par les passions identitaires et les réflexes autoritaires qui gagnent autant les élites dominantes que les citoyens ordinaires et qui interrogent sur le sens profond que nous donnons aujourd'hui au mot « démocratie».

Marseille, le 25 mars 2019

32. "Les gilets jaunes sans les Français issus de l'immigration" [En ligne], Boulevard Voltaire.fr, 10 février 2019, http://www.bvoltaire.fr/les-gilets-jaunes-sans-les-francais-issus-delimmigration/.

33. GUILLEMAIN, Jacques, "Gilets jaunes, battez-vous contre l'immigration, pas contre les riches !" [En ligne], Riposte läque.com, 9 février 2019, https://ripostelaique.com/giletsjaunes-battez-vous-contre-limmigration-pas-contre-les-riches.html ; CYRANO, "Le silence des 'chefs' gilets jaunes sur l'immigration fait le jeu de Macron" [En ligne], Riposte laïque.com, 15 février 2019, https://ripostelaique.com/le-silence-des-chefs-gilets-jaunessur-limmigration-fait-le-jeu-de-macron.html. 